\title{
Cultivos urbanos en un aula de matemáticas inclusiva ${ }^{1}$
}

\author{
Urban farming in inclusive mathematics classroom
}

A agricultura urbana na inclusive aula de matemática

Recibido: mayo de 2013

Aceptado: agosto de 2013
Jaison Fernando Ariza Ardila ${ }^{2}$

JeimmyLizeth Bernal Calcetero ${ }^{3}$

Maria Camila Rubiano Salamanca ${ }^{4}$

Yina Paola Salamanca Monroy

\section{Resumen}

En el presente trabajo se describe un proyecto de aula en curso - en un aula inclusiva-, el cual hace referencia a la realización de cultivos urbanos, este trabajo se describe en 4 fases en las cuales se desarrollan conceptos y nociones matemáticas.

Palabras clave: Proyecto de aula; matemáticas y otras disciplinas; cultivos urbanos; pensamientos matemáticos; alumno; diversidad; inclusión.

\section{Abstract}

This paper describes an ongoing classroom project - in an inclusive classroom, which refers to the realization of urban farming, this work is described in four phases in which concepts and notions develop math.

Keywords: Classroom project, mathematics and other disciplines, urban farming, mathematical thinking, student, diversity, inclusion.

\section{Resumo}

Este artigo descreve um projeto de sala de aula em curso - em uma sala de aula inclusiva, que se refere à realização da agricultura urbana, este trabalho é descrito em quatro fases em que os conceitos e noções de matemática desenvolvem.

Palavras-chave: Projeto Sala de Aula, matemática e outras disciplinas, a agricultura urbana, pensamento matemático, estudante, diversidade, inclusão.

1 Artículo de Investigación.

2 Universidad Distrital Francisco Jose de Caldas. Bogotá, Colombia. Contacto: jaison.punk@hotmail.com

3 Universidad Distrital Francisco Jose de Caldas. Bogotá, Colombia. Contacto: cabelije@hotmail.com

4 Universidad Distrital Francisco Jose de Caldas. Bogotá, Colombia. Contacto: camiiya1991@hotmail.com

5 Universidad Distrital Francisco Jose de Caldas. Bogotá, Colombia. paito-48@hotmail.com Contacto: 


\section{Contextualización}

El presente trabajo está siendo desarrollado en el espacio académico de Práctica Intensiva por estudiantes de la Universidad Distrital de decimo semestre, es un proyecto de aula titulado "Agricultura Urbana", en este se desarrollan los cinco pensamientos matemáticos propuestos por el Ministerio de Educación Nacional, presentados en los lineamientos y estándares, además de una reflexión y acción por parte de los estudiantes de los grados 702 y 703 y de los docentes, en la institución IED OEA, la cual, está ubicada en la localidad de Kennedy cuyo estrato oscila entre el dos y el tres. Esta institución hace parte de una red de instituciones planteadas en el Plan Sectorial de Educación, Bogotá Una Gran Escuela (2004-2008), en el cual su eje central es la estrategia de Inclusión social y Protección a la Niñez en la escuela, además se busca que en las escuelas se reconozcan y valoren la diversidad cultural de la población estudiantil por medio de prácticas de enseñanza, planes de estudio y ambientes de aprendizaje diseñados a partir de estas diferencias.

Como se afirmó anteriormente es una institución de inclusión, es decir, asisten a esta estudiantes de aulas regulares y estudiantes en condición de discapacidad visual. Por tanto, es una tarea ardua del docente estar en buscar estrategias que permitan, que los estudiantes en su totalidad logren desarrollar habilidades, en diferentes áreas, específicamente las matemáticas. Se pretende lograr esto por medio de una articulación entre el proyecto de agricultura urbana, la matemática escolar y la inclusión.

Específicamente se trabaja con 80 estudiantes de grado séptimo cuyas edades están comprendidas entre los 11 y 12 años, entre ellos se encuentra únicamente dos estudiante con limitación visual, Angie Herrera cuyo diagnóstico es baja visión y Laura Silva cuyo diagnóstico es pérdida total de la visión.

\section{Referentes teóricos}

Para el desarrollo de los referentes se tienen en cuenta tres aspectos teóricos, el primero referido a los conceptos y nociones matemáticas escolares que se han utilizado, el segundo se refiere a la inclusión en la escuela y por último el aspecto metodológico. Los cuales se desarrollaran a continuación:

Matematico: Teniendo en cuenta que se realiza una articulación entre los cinco pensamientos matemáticos, se presentan los temas específicos a trabajar y su respectiva articulación con los pensamientos, dándole mayor prioridad al pensamiento numérico y sistemas numéricos y el pensamiento geométrico

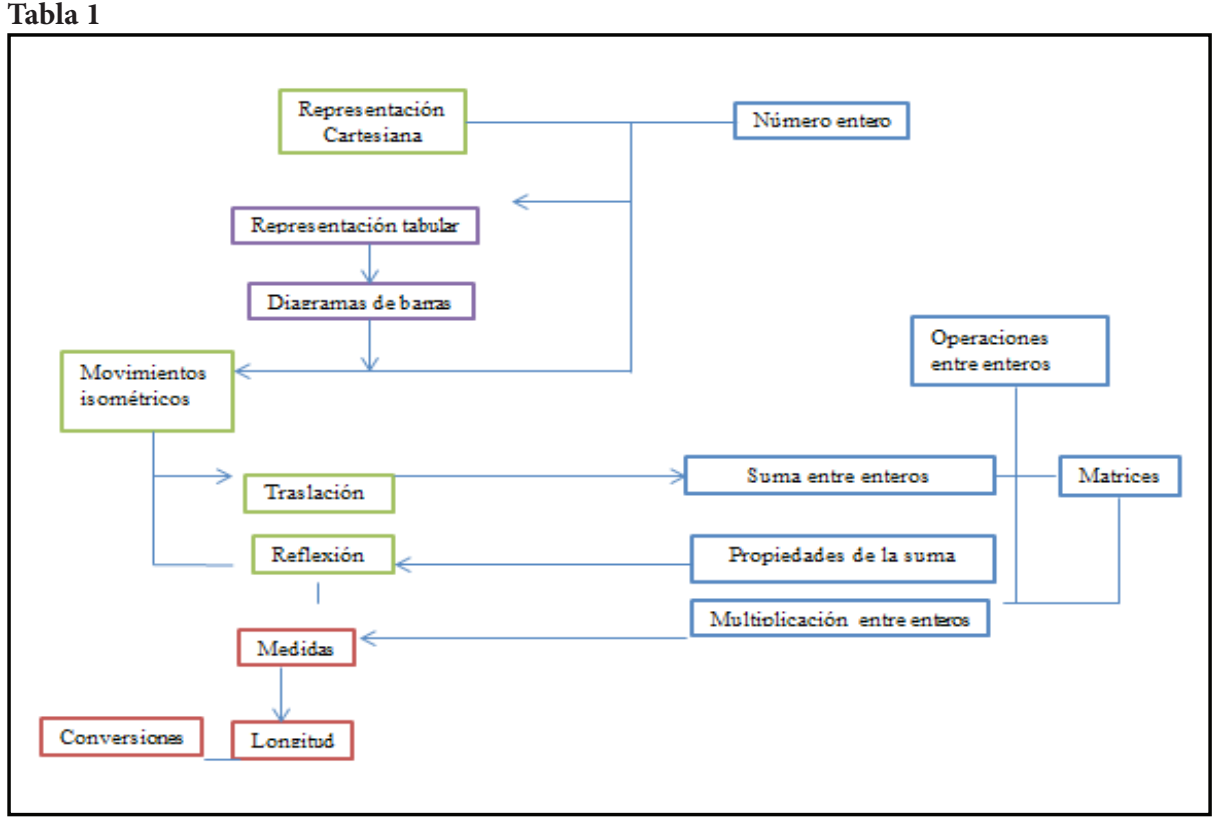

Fuente: elaboración propia 
Inclusion: Se habla de inclusión y no de integración pues se piensa que la inclusión plantea mejores oportunidades para los estudiantes y el contexto en general donde se pretende llevar a cabo este proceso, según Lübeck \& Rodrigues (2013) la inclusión propone cambios sustanciales en la sociedad y las escuelas, de modo que todas las personas con diferentes condiciones físico-biológico-sensoriales pueden tener la mismas oportunidades que, las demás personas, respetando y valorando las diferencias en cualquier ambiente. Estos ambientes inclusivos nos permiten respetar principios fundamentales, como la solidaridad, la cooperación, el diálogo simétrico etc, y nos permite repensar la matemáticas proponiendo a los docentes el reto de ser formadores matemáticos.

Según la UNESCO 1994." Aquellos alumnos con necesidades educativas especiales deben tener acceso a los colegios de educación regular, donde deben ser acomodados con estrategias pedagógicas centradas en el alumno para responder a sus necesidades". Por tanto como se adapta todo el material con el que se trabajara en clase.

Metodologico: El proyecto de aula permite buscar alternativas para que los estudiantes se motiven e involucre con el aprendizaje de las matemáticas según León citando a Rodriguez (2001) la pedagogía por proyectos permite fundamentalmente insertar la escuela en la vida, cristalizar los interés de los estudiantes frente al conocimiento y dejar de atomizar los contenidos escolares y los procesos de aprendizaje para responder a aprendizajes significativos y a la solución de problemas que plantean en el proyecto de aula.

Para llevar a cabo un proyecto de aula Según Leon (s.f) se proponen tres principios: - La explicación: Está asociada a un diseño didáctico y exige que los diseños para el aula y las acciones didácticas obedezcan a factores asociados con necesidades de formación. En este principio se desarrolla la estructuración de cada uno de los proyectos, pues cada uno de ellos debe responder a una compresión de cómo se puede desarrollar el proceso enseñanza y aprendizaje en el marco de las matemáticas. - La realización: Implica la naturaleza de la misma acción didáctica: ser realizable y realizarse para que se efectué como acción, por tanto se exige la obtención de productos definidos en cada una de las fases y condiciona las formas de interacción entre profesores y estudiantes en relación la elaboración de conocimiento en el aula, con el uso de instrumentos y con el desarrollo de una seria de acciones intencionada y orientadas. Y por último - De proyección: Toda acción didáctica necesariamente tendrá efectos, estará intencionada para que ocurran proceso y espera efectos, estará intencionada para que ocurran procesos y esperar obtener resultados inmediatos, mediatos de producto y procesos, en el aula, la cultura institucional en general la familia y la sociedad.

\section{Descripción de la experiencia:}

Para el desarrollo de este proyecto se elaboran tres fases (planeación, cultivando y reflexión) la cuales se describen a continuación:

Fase I: PLANEACION: Esta responde a la pregunta del ¿Qué y donde cultivamos? En esta fase los estudiantes hacen un estudio geográfico y climático empezando por lo general (el mundo), para pasar a lo particular (Mi país y Mi ciudad). Con esto ellos logran afirmar que tipo de productos agrícolas se pueden cultivar dando afirmación matemáticas referidas a el numero relativo, el numero entero, puntos de referencia y ubicación geográfica por medio de plano cartesiano.

Foto 1: Herbarios. Algunos resultados de la I fase.

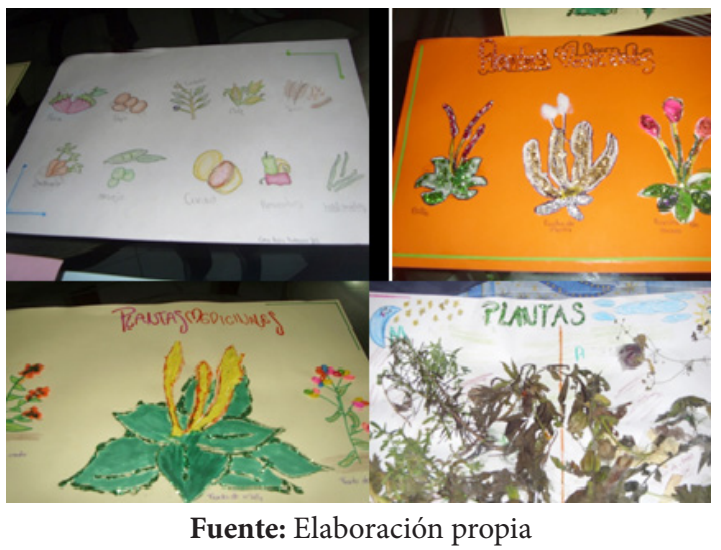

Talleres realizados: Taller 1: Viajando por el mundo Taller 2: El clima de mi país no es así porque sí. Taller 3: ¿Y mi ciudad que clima toca? Taller 4: Que puedo cultivar? 
Fase II: CULTIVANDO: En esta fase se pretende que los estudiantes hagan planos sobre el terreno donde van a cultivar y por medio de herramientas matemáticas (escalas, conversiones, transformaciones geométricas por medio de matrices) logren organizar de la manera más favorable este espacio, mientras en la clase de matemáticas se trabajan conceptos matemáticos; paralelamente se trabajara en la construcción de las camas. Esta fase no se ha culminado por tanto los estudiantes no han realizado los planos y la preparación de las cama está en proceso.

Foto 2: Medición y preparación de Camas SEQ. Arabic: Resultados preliminares fase II
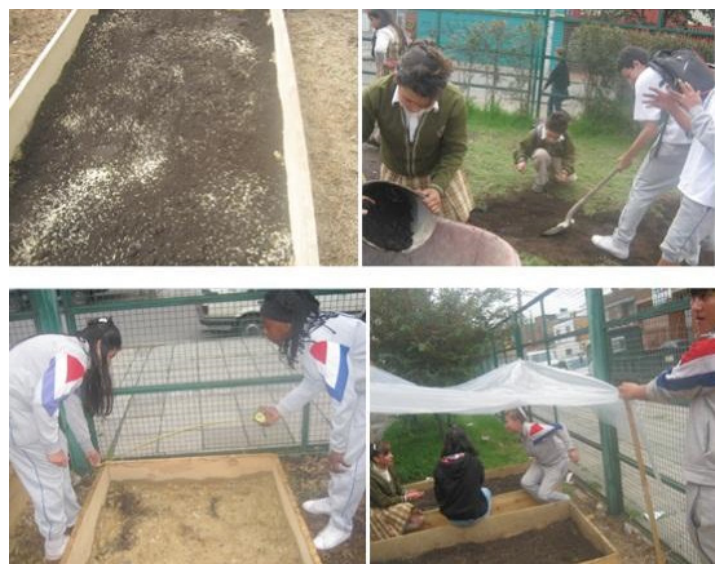

Fuente: Elaboración propia

Talleres trabajados: Taller 5: Organizando para cultivar. Taller 6: Aquí cultivaremos.

Talleres a trabajar: Taller 7: Cuanto nos costara cultivar. Taller 8: El valor de la tierra. Taller 9: Sembrando vamos

\section{Logros y dificultades}

Lo que se pretendía en esta fase era hacer un estudio del clima, para saber qué es lo que podemos cultivar en nuestra granja escolar, por medio del trabajo con los números enteros, permitiendo un acercamiento a los estudiantes a la comprensión de estos números. Según Borjas (2009) el aprendizaje del concepto de número está ligado al desarrollo de habilidades, destrezas y conceptualizaciones en aspectos tales como: Sentido de número y estimación;
Conocimiento de los múltiples usos de los números; El conteo y las estrategias para operar a través del conteo. Las cuales los estudiantes trabajaron por medio de la ubicación geográfica, relacionándolo con el plano cartesiano, con esto comprendieron, los ejes de referencia, organización del plano (signos de los cuadrantes), la importancia de la escritura de las parejas ordenas. Además de esto lograron encontrar característica del plano cartesiano (rectas paralelas y perpendiculares).

En el pensamiento geométrico, los estudiantes lograron inicialmente acercarse por medio de la manipulación de recursos como, hojas mantequilla y el caleidoscopio a los movimientos rígidos (traslación y reflexión) para posteriormente asociarla a representación cartesiana y matricial y con esto realizar operaciones (suma, resta, multiplicación) entre enteros.

En el pensamiento aleatorio y sistemas de datos, los estudiantes lograron analizar diferentes datos referidos al clima de Bogotá y ejemplos de algunos cultivos, también pasaron de representaciones graficas a tabulares.

\section{Reflexión}

El uso de materiales manipulativos, ayuda a los estudiantes a comprender mejor nociones matemáticas, pero hay que tener cuidado con la planeación de estos, ya que para Laura, este recurso manipulativo, tuvo beneficios pero también inconvenientes, ya que la lana y pegarla con cinta, hacía que esta no tuviera una rigidez, que representara una línea recta.

Además del material, la gestión del docente debe tener en cuenta su manera de explicar, teniendo en cuenta que está en un aula inclusiva. En esta medida se encuentra dificultad, dado que en ocasiones no se hace uso de un buen lenguaje, que permita la inclusión.

Respecto al proyecto de aula, con esta actividad se ha permitido vincular el concepto de paralelismo con las ciencias sociales. Pero se debe tener cuidado para no perder la mirada del énfasis en matemáticas. 
El proyecto agricultura urbana permite articular de una manera eficiente los pensamientos matemáticos y vincular la matemáticas a diferentes áreas del conocimiento (sociales, ciencias naturales), también nos permitió acerca a los estudiantes al trabajo del campesino.

Respecto a la experiencia de los cultivos y los estudiantes con limitación visual no en todos los casos fue favorable, puesto que ha una de los estudiantes el acudiente no le permitió acudir a las sesiones de práctica, argumentando que la estudiante de 703 podía correr algún riesgo. Por otra parte la estudiante de 702 con limitación visual asistió a la práctica del cultivo, realizando varias de las tareas del día (filtrar tierra, abonar, sembrar) alternando las labores con sus demás compañeros y socializando de una manera colaborativa, solidaria, respetuosa y divertida.

\section{Referencias}

Borjas, D.(2009 ). Aprendizaje de los números enteros una "experiencia significativa" en estudiantes de séptimo grado de la escuela nacional de música (Tesis de maestría). Universidad Nacional Pedagógica Francisco Morazán.

León, L. Rocha, P \& Vergel, R (s.f.). El juego, la resolución de problemas y el proyecto de aula como dispositivos en las didácticas de la matemática y de la estadística. Bogotá

Lübeck, M. \& Rodrigues, T. D.(2013). Incluir é Melhor que Integrar: uma concepção da Educação Etnomatemática e da Educação Inclusiva. Revista Latinoamericana de Etnomatemática. http://www.revista.etnomatematica.org/index.php/RLE/article/ view/71/64\# consultado 11/08/2013. 